〈Research Paper〉

\title{
Process Balance of Natural Indigo Production based on Traditional Niram Method
}

\author{
Younsook Shin ${ }^{\dagger}$, Dong II Yoo $^{1}$ and Kangwha $\mathrm{Kim}^{2}$ \\ Department of Clothing and Textiles/Human Ecology Research Institute, Chonnam National University, \\ Gwangju, Korea \\ ${ }^{1}$ Department of Polymer and Fiber System Engineering, Chonnam National University, Gwangju, Korea \\ ${ }^{2}$ Department of Food and Nutrition, Chonnam National University, Gwangju, Korea
}

(Received: November 29, 2012 / Revised: December 12, 2012 / Accepted: December 13, 2012)

\begin{abstract}
In this work, the natural indigo production process from Polygonum tinctorium was balanced based on the traditional Niram method in Korea. A standard procedure was determined considering the conditions of indican extraction from plant material, the amount of alkali for precipitation, storage of extract, etc. The effect of experimental conditions on the yield of crude dye was investigated. The contents of indigo and indirubin of the crude dyes were analyzed by HPLC. Increase of the amount of crude dye was observed within 1-2.5 days of extraction time. Longer extraction beyond 2.5 days resulted in a slight decrease in the amount of crude dye. There was no consistency in terms of indigo content depending on extraction $\mathrm{pH}$. We found that the storage of extract or harvested plants affected adversely to dye yield and dye quality. Based on the lab scale extraction, large scale extraction was performed for 2-2.5 days in water and 2.0-2.5 g/L of $\mathrm{Ca}(\mathrm{OH})_{2}$ was applied for precipitation of indigo dye. We obtained natural indigo dye containing about $15 \%$ of pure indigo in scale-up production using whole plant except root.
\end{abstract}

Keywords: Polygonum tinctorium, Niram, indican, indigo, indirubin

\section{Introduction}

Natural indigo is the most important blue dye for textile dyeing with natural dye. At current market, indigo is used mainly for dyeing cotton yarn to produce denim. With the worldwide issue for sustainability and the demand for natural eco-products, there has been a revival of natural dyes. Although various shades, such as red, yellow, brown, gray and so on, can be obtained from various sources of plants, there is no practical plant resource for blue shade ${ }^{1,2}$. So, indigo is needed persistently as a source of blue color. The synthetic indigo produced annually is estimated as much as 22 thousand tons. There have been many researches to replace the chemical synthesis of indigo dye by applying biotechnology ${ }^{3-6)}$. On the other hand, efficient ecological production of indigo based on simple extraction method has been also performed by European researchers ${ }^{7,8}$.

In eastern Asia, main indigo producing plant species

${ }^{\dagger}$ Corresponding author: Younsook Shin (yshin@jnu.ac.kr) Tel.: +82-62-530-1341 Fax.: +82-62-530-1349

(C)2012 KSDF 1229-0033/2012-12/253-259 is Polygonum tinctorium, a subtropical annual plant ${ }^{9,10)}$. Natural indigo is normally obtained from cultivated species by water extraction and the following alkali precipitation. In Korea, there have been two different ways of traditional indigo dyeing, i.e., the Banmul method and the Niram method. The former is the dyeing from an indigo extract by water directly in summer season and the latter is the dyeing by using an indigo-alkali complex available for all season. The latter has some advantages of getting deeper color and can be stored and used without seasonal limitation. In the Niram method, indican is extracted by steeping the plant leaves and stems for several days. As the second step of this method, the powder of oyster shell or shellfish is added onto the extract while beating for the introduction of air oxygen as much as possible until blue indigo precipitated, and then left it sunken. The sediment is collected and filtered through a cotton fabric. Thick paste obtained is called Niram, which means indigo mud. This traditional method has been used successfully in forming natural indigo, but quality and quantity of the dye is varied every time 
because the conditions for extraction and subsequent conversion into indigo have been relied on the experience of individual dyers. So, it is necessary to find more reliable process for producing relatively consistent quality of dye.

The objective of this study is to investigate the effect of process variables systematically to improve the disadvantages of traditional method. Based on the traditional Niram method, the process was balanced to get maximum indigo yield and indigo contents with less energy consumption.

\section{Experimental}

\subsection{Indigo plants}

Indigo seeds were sowed at the end of March in Naju (Chonnam Province, southwest of Korea) and indigo plants were harvested during July and August, 2008.

\subsection{Extraction and crude indigo dye preparation}

To obtain optimal extraction condition, lab scale extraction was carried out. Only leaves $(50 \mathrm{~g})$ were steeped in distilled water $(1 \mathrm{~L})$ at $25-26^{\circ} \mathrm{C}$. The extraction was made by maintaining leaves in distilled water at various time (1, 2, 2.5 and 3 day). For comparison to the extraction in distilled water $(\mathrm{pH} \mathrm{6})$, the extraction also was performed at $\mathrm{pH} 3.5$, adjusted by using acetic acid. $\mathrm{Ca}(\mathrm{OH})_{2}$ was added in filtered extracts with stirring for $20 \mathrm{~min}$ using a homogenizer, and left it overnight for sedimentation. Clear supernatant was siphoned off and then the sediment in slurry was dried in a convection oven at $50^{\circ} \mathrm{C}$ and weighed. The dried sediment was finely ground and used for analysis. In lab scale extraction, $\mathrm{Ca}(\mathrm{OH})_{2}$ and $\mathrm{NaOH}$ was used of analytical grade.

\subsection{Scale-up extraction}

Indigo plants were harvested for large scale production of indigo dye on August, 2008. Into a $1000 \mathrm{~L}$ stainless steel tank, ground water of $27^{\circ} \mathrm{C}$ was poured over the plants with leaves and stems (80
$\mathrm{Kg}, 10 \mathrm{~L}$ of water to $1 \mathrm{Kg}$ of plant materials). The steeped plants were weighted down with 6 stoneblocks (weighing $5 \mathrm{Kg}$ each) to hold plants under water to exclude air for maintaining anaerobic condition. After steeping for 2.5 days, the extract (790 L) was pumped into three settling tanks and added different amount of $\mathrm{Ca}(\mathrm{OH})_{2}(1.5,2.0,2.5$, and 3.0 $\mathrm{g} / \mathrm{L})$ in each tank respectively, and aerated for 30 min by using a compressor to help oxidation of indigo precursors and precipitation of indigo. The indigo was settled down for $12 \mathrm{hrs}$ and the supernatant was removed. The sediment was collected and filtered through layers of a cotton fabric. After drying in a vacuum oven at $50^{\circ} \mathrm{C}$, the amount of crude indigo dye was weighed and pulverized for analytical tests and dyeing experiments. $\mathrm{Ca}(\mathrm{OH})_{2}$ was used of industrial grade.

\subsection{Indigo and indirubin quantification}

Indigo and indirubin contents were determined according Liau et al. ${ }^{11)}$. HPLC analysis was carried out on an Agilent 1200 liquid chromatography system (Agilent technologies Inc., Waldbronn, Germany) equipped with two pumps, UV detector and Rheodyne injector (50mL loop). Chromatographic conditions were used as follows: The LC column was a Zorbax Eclipse XDB-C18 (4.5 mm $\times 150 \mathrm{~mm}, 5 \mathrm{~m})$ (Palo Alto, CA, USA). Two mobile phases $\mathrm{A}$ and $\mathrm{B}$ were filtered through a $0.45 \mathrm{~m}$ filter and run at flow rate of $1.0 \mathrm{~mL} / \mathrm{min}$ and room temperature of $25^{\circ} \mathrm{C}$. Mobile phase A consisted of water with $0.1 \%$ trifluoroacetic acid (TFA) and mobile phase $\mathrm{B}$ was acetonitrile (ACN) containing $0.1 \%$ TFA. A linear gradient was maintained from $40 \% \mathrm{~B}$ to $85 \% \mathrm{~B}$ for $15 \mathrm{~min}$.

Indigo component was eluted at $9.18 \mathrm{~min}$, showing peaks at 280, 610, and 550nm. Whereas indirubin was eluted at $10.02 \mathrm{~min}$, showing peaks at 280 and $550 \mathrm{~nm}$. They were well separated and all the peaks had good shape. For the quantification of indigo and indirubin contents of the prepared indigo dye, the peak at $280 \mathrm{~nm}$ was used because the peak signal was strong and also it was a common peak for two components. The peak areas at $280 \mathrm{~nm}$ were determined 
and calculated the concentrations of indigo and indirubin, respectively, by using the standard calibration curves $^{12)}$.

The measurement of each sample was tried and averaged in triplicate. Synthetic indigo (Vat. Blue 1, Aldrich) and indirubin (Alexis Biochemical, USA) were used of analytical grade as standard dyestuffs for calibration.

\section{Results and Discussion}

\subsection{Effect of process variables on dye yield and indigo content}

Traditional Niram method was modified by using calcium hydroxide instead of oyster shell powder and dried the Niram of mud-like form into a crude dye of powder form. Some process variables, such as extraction time and $\mathrm{pH}$, storing time after extraction, and calcium hydroxide concentration, were applied to optimize conditions in lab scale and then applied to scale-up production.

The mechanism of natural indigo formation is known very well. Indicans present in the plants are converted by enzyme ( $\beta$-glucosidase) present in the leaf tissues to indoxyl and glucose. Two indoxyl molecules are combined by oxygen in air to form indigo and also indirubin in side reaction (Figure 1).

The yield of crude dye and indigo content plotted for extraction time and the amount of alkali $\left(\mathrm{Ca}(\mathrm{OH})_{2}\right)$ used in the precipitation step (Figure 2, 3). Total mass of crude dye obtained was dependent on extraction time and the concentration of $\mathrm{Ca}(\mathrm{OH})_{2}$.
The crude dye obtained is a mixture of indigo and calcium hydroxide. Dye quality in terms of indigo content would be different with treatment conditions. Increase of the amount of crude dye was observed within 1-2.5 days of extraction time.

Longer extraction beyond 2.5 days resulted in a slight decrease in the amount of crude dye (Figure 2). It is speculated that enzyme ( $\beta$-glucosidase), which converts indican to indoxyl, lost its activity in longer extraction environment and alkaline condition, resulting in the decrease of indigo formation. Maugard et al. reported that the optimum $\mathrm{pH}$ of $\beta$-glucosidase for indican hydrolysis was 3 and the activity of $\beta$-glucosidase was dimished as extraction time got longer ${ }^{4)}$. The quantity of crude dye was increased with the concentration of $\mathrm{Ca}(\mathrm{OH})_{2}$. But indigo content in the crude dye ( $\%$ indigo/g of crude dye) decreased with the increase of the concentration of $\mathrm{Ca}(\mathrm{OH})_{2}$, irrespective of extraction time (Figure 3).

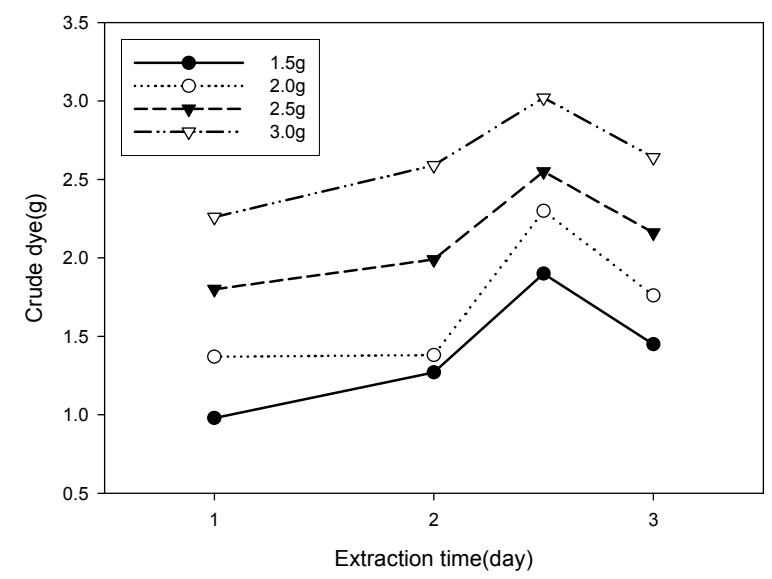

Figure 2. Effect of extraction time on the amount of crude dye depending on $\mathrm{Ca}(\mathrm{OH})_{2}$ concentration.

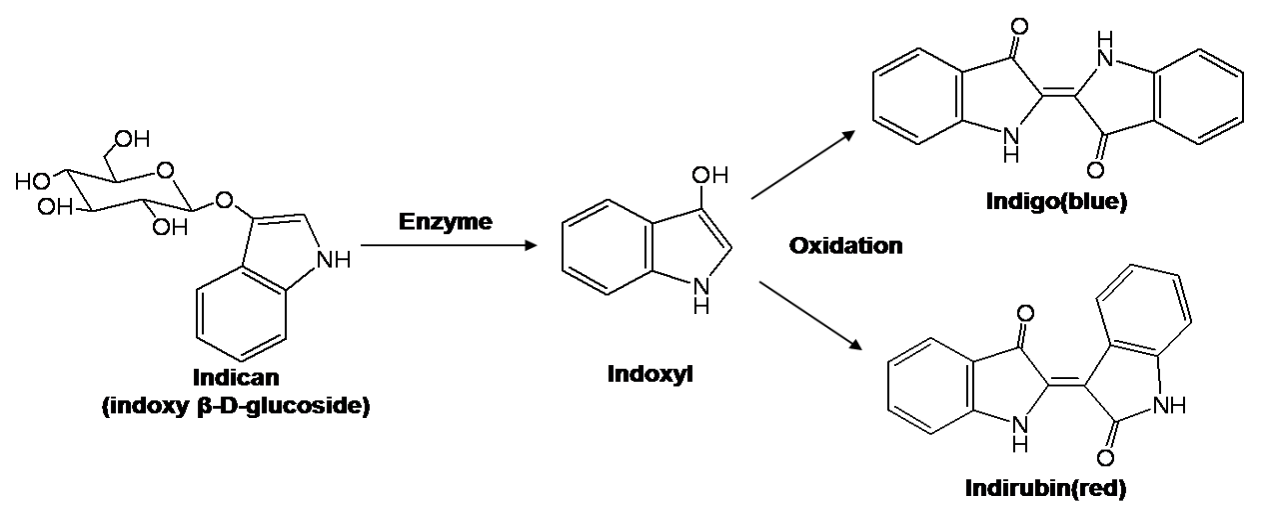

Figure 1. The formation of indigo and indirubin from indican. 


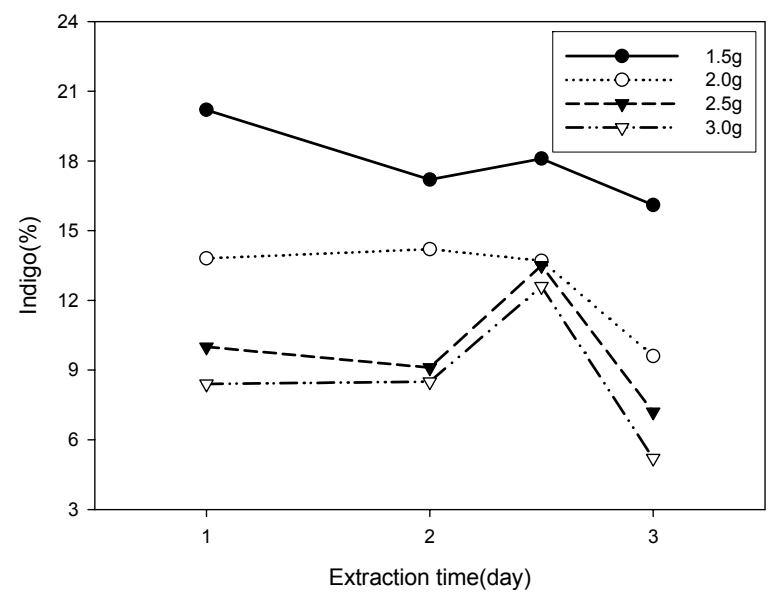

Figure 3. Effect of extraction time on indigo content depending on $\mathrm{Ca}(\mathrm{OH})_{2}$ concentration.

This is because the extracted solution for the same extraction time contained the same quantity of indican, that is the same amount of indigo formed, and as a result, lower indigo content value when more $\mathrm{Ca}$ $(\mathrm{OH})_{2}$ was added. Based on the obtained results, 2.5 days of extraction time was considered as appropriate.

Extraction $\mathrm{pH}$ affected to extraction efficiency differently depending on extraction time as shown in Figure 4 and 5. For 1 day extraction, more efficient extraction was led to higher yield of crude dye at $\mathrm{pH}$ 6.0, compared to extraction at $\mathrm{pH} 3.5$. However, when extraction time extended for 2 days, the influence of $\mathrm{pH}$ became marginal. Ratio of indigo contained in crude dye got lower as the amount of $\mathrm{Ca}(\mathrm{OH})_{2}$ used got more for precipitation. There was no consistency in terms of indigo content depending on extraction $\mathrm{pH}$. It is considered that the adjustment of $\mathrm{pH}$ for extraction is not necessary. Also, considering environmental and economical point of view, the addition of chemicals should be avoided during extraction.
As extracts were stored for $36 \mathrm{hrs}$, the results of the yield of crude dye and indigo contents are given in Table 1. Severe decrease in crude dye yield within $36 \mathrm{hrs}$ of storage at room temperature was resulted. And decrease in indigo content was also observed as storing extracts. We found that the storage of extract or harvested plants affected adversely to dye yield and dye quality.

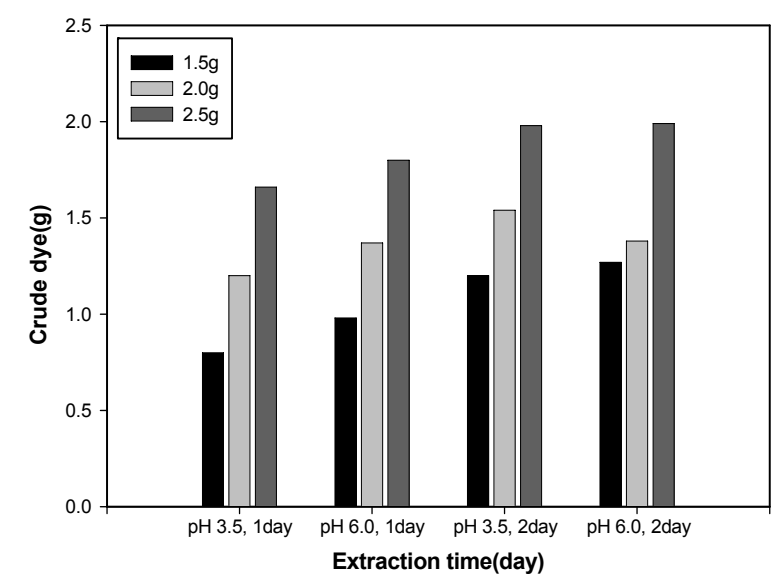

Figure 4. Effect of $\mathrm{pH}$ on crude dye yield.

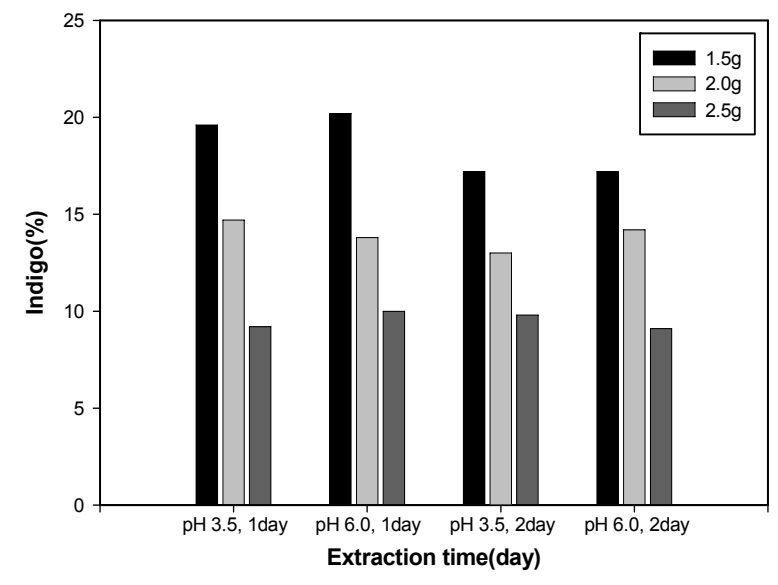

Figure 5. Effect of $\mathrm{pH}$ on indigo content.

Table 1. Comparison of dye quality depending on storing extracts

\begin{tabular}{ccccc}
\hline Extraction pH & Storage time (hr) & Crude dye (g) & Indigo (\%) & Indirubin (\%) \\
\hline \hline \multirow{2}{*}{3.5} & 0 & 1.25 & 21.7 & 0.000 \\
& 36 & 0.95 & 8.3 & 0.089 \\
\hline \multirow{2}{*}{6.0} & 0 & 1.00 & 17.8 & 0.005 \\
& 36 & 0.84 & 10.8 & 0.081 \\
\hline
\end{tabular}

1day extraction, $2.0 \mathrm{~g} / \mathrm{L} \mathrm{CaOH}_{2}$ 
In the case of using extracts immediately after one day extraction at acidic condition $(\mathrm{pH} 3.5)$, indirubin was not detected by HPLC analysis. With the extracts stored for $36 \mathrm{hrs}$, indirubin was detected in the crude dye even if its concentration was very low. The factors for influencing indirubin formation are not completely investigated, but weak alkaline condition and elevated temperature tend to favor its formation".

Keeping extracts for $36 \mathrm{hrs}$ decreased crude dye yield as much as $16-24 \%$ and also lowered indigo content as much of $7-13.4 \%$. On the basis of results, it is recommended that extract solution should be used without any delay after finishing extraction.

\subsection{Scale-up production}

On the basis of the results obtained from small scale extraction, the production was scaled up in laboratory as well as in a farm-mill near indigo plant cultivation field. We used leaves only in laboratory production, while used the plants including leaves and stems in a farm-mill in Naju. Based on the lab scale extraction, scale-up extraction was performed for 2-2.5 days and $2.0 \mathrm{~g} / \mathrm{L}$ of $\mathrm{Ca}(\mathrm{OH})_{2}$ and the results of scale-up production are given in Table 2. Yield in Table 2 represents that the ratio of pure indigo produced from plants.

It was reported that indigo yield is affected by light radiation and water, thereby leaf production per plant ${ }^{8,13,14)}$. A higher yield and better quality of dye was obtained by a washing leaves before extraction. Washing increased indigo content as much as $35 \%$, as shown in Table 2. Impurities such as soil influenced indigo formation, but repeated washings adversely affected dye yield. Repeated washing may remove native enzyme which related to indigo production and adversely affect to indigo formation ${ }^{4)}$.
It is considered that storage time and pretreatment (washing) are important factors for better quality of dye. But, a washing process of large quantity of plants after harvest would be impossible and water consumption during process should be taken into account very carefully from environmental viewpoint. Indigo dyes produced from whole plant except root in the scale-up study have consistent quality of dye with about $15 \%$ of indigo content.

Using only leaves, yields of pure indigo were obtained $0.51 \%$ without washing and $0.81 \%$ with washing, meaning that pure indigo can be produced about $51-81 \mathrm{~g}$ from $10 \mathrm{Kg}$ of leaves. Using leaves and stems except roots, yield was $0.354 \%$, meaning that $35.4 \mathrm{~g}$ of pure indigo from $10 \mathrm{Kg}$ of whole plant except root. We harvested about $600 \mathrm{Kg}$ of leaves and stems from a field of $660 \mathrm{~m}^{2}, 2.12 \mathrm{Kg}$ of pure indigo can be obtained by calculation. Crop growth can change every year depending on climate conditions including temperature and rainfall, thereby dye yield and indigo content could be affected. The results of this study are not definite, but they will fluctuate by nature.

For comparing the quality of indigo dye produced in this study, we analyzed some imported natural indigo dyestuffs in current market and the results are given in Table 3.

The quality of natural indigo products were very wide depending on imported origin country.

Concentration of indigo precursor in indigo plants is varied by plant species and also, production process is different depending on regional culture. China produced natural indigo from Poligonum tinctorium as same as in Korea, while in India, Indigofera tinctoria is cultivated. In Europe, natural indigo is produced from Isatis tinctoria (woad) ${ }^{8}$.

Table 2. Scale-up production

\begin{tabular}{lccccc}
\hline \multicolumn{1}{c}{ Plant } & Amt. (Kg) & Crude dye (g) & Indigo (\%) & Indirubin (\%) & Yield (\%) \\
\hline \hline Leaves & 2.8 & 85 & 16.79 & 0.33 & 0.510 \\
Leaves $^{\mathrm{a}}$ & 27.8 & 995 & 22.59 & 0.34 & 0.810 \\
Leaves+Stems & 243.0 & 5515 & 15.44 & 0.75 & 0.354 \\
\hline a:
\end{tabular}


Table 3. Quality of imported natural indigo

\begin{tabular}{cccccc}
\hline Country & Indigo (\%) & Indirubin (\%) & Country & Indigo (\%) & Indirubin (\%) \\
\hline China-1 & 14.3 & 0.52 & France-1 & 21.4 & 0.13 \\
China-2 & 4.4 & 0.21 & France-2 & 63.3 & 1.33 \\
China-4 & 12.8 & 0.33 & Germany & 12.8 & 0.08 \\
India-1 & 96.0 & 0.14 & & & \\
India-2 & 87.2 & 0.18 & & & \\
\hline
\end{tabular}

Dyestuffs imported from India showed the highest quality in terms of indigo content. It has been known that Indigofera tinctoria produces superior quality of indigo dyestuff ${ }^{7}$. It is thought that the cultivation of Indigofera tinctoria should be tried in Korea because the weather seems to be shifted to warmer temperature recently. It could be grown in the southern part of Korea which is subtropical zone.

\section{Conclusion}

Based on the results from the lab scale extraction, large scale extraction in a farm mill was carried out for 2-2.5 days extraction in water and 2.0-2.5 g/L of $\mathrm{Ca}(\mathrm{OH})_{2}$ was applied for precipitation. We obtained natural indigo containing about $15 \%$ of pure indigo in scale-up production using whole plant except root, which was comparable product in quality with natural indigo made in lab scale. This study will provide a standard procedure for natural indigo production process based on traditional Niram method. The procedure will improve the reproducibility of indigo dye products and better quality of the dyed products.

\section{Acknowledgement}

This work was supported by the National Research Foundation of Korea(NRF) grant funded by the Korea government(MEST) (No. 2012015736).

\section{References}

1. M. W. Huh, Dyeability and Functionality of Cotton Fabrics Treated with Persimmon Juice, Textile Coloration and Finishing(J. Korean Soc. Dye. and Finish.), 23(4), 241(2011).
2. S. W. Nam and K. T. Kim, Preparation and Screen Printing of Natural Dye Powders, Textile Coloration and Finishing(J. Korean Soc. Dye. and Finish.), 22(4), 314(2010).

3. J. Y. Kim, J. Y. Lee, Y. Shin, and G. J. Kim, Characterization of an Indican-hydrolyzing Enzyme from Sinorhizobium meliloti, Process Biochemistry, 45, 892(2010).

4. T. Maugard, E. Enaud, A. L. Sayette, P. Choisy, and M. D. Legoy, Beta-Glucosidase-catalysed Hydrolysis of Indican from Leaves of Polygonum tinctorium, Biotechnology Progress, 18(5), 1104 (2002).

5. Y. Minami, Y. Shigeta, U. Tokumoto, Y. Tanaka, K. Yonekura-Sakakibara, and H. Ohoka, Cloning, Sequencing, Characterization, and Expression of a Beta-glucosidase cDNA from the Indigo Plant, Plant Science, 142, 219(1999).

6. Y. Minami, H. Takao, T. Kanafuji, K. Miura, M. Kondo, and I. H. Nishimura, Beta-Glucosidase in the Indigo Plants: Intracellular Localization and Tissue Specific Expression in Leaves, Plant Cell Physiology, 38, 1069(1997).

7. T. Bechtold, A. Turcanu, S. Geissler and E. Ganglberger, Process Balance and Product Quality in the Production of Natural Indigo from Polygonum tinctorium Ait. Applying Low-technology Methods, Bioresource Technology, 81, 171(2002).

8. E. Camperol, L. G. Angelini, S. Tozzi, and M. Bertolacci, Seasonal Variation of Indigo Precursors in Isatis tinctoria L. and Polygonum tinctorium Ait. As affected by Water Deficit, Environmantal and Experimental Botany, 58, 223(2006). 
9. D. Cardon, "Natural Dyes: Sources, Tradition, Technology and Science", Archtype Publications Ltd., London, p.339, 2007.

10. K. G. Gilbert, H. G. Maule, B. Rudolph, M. Lewis, H. Vandenburg, E. Sales, S. Tozzi, and D. T. Cooke, Quantative Analysis of Indigo and Indigo Precursors in Leaves of Isatis spp. and Polygonum tinctorium, Biotechnology Progress, 20, 1289(2004).

11. B. C. Liau, T. T. Jong, M. R. Lee, and S. S. Chen, LC-APCI-MS Method for Detection and Analysis of Tryptanthrin, Indigo, and Indirubin in Daqingye and Balangen, J. Pharmaceutical and Biomedical Analysis, 43, 346(2007).
12. Y. Shin, K. Son, and D. I. Yoo, Tencel Dyeing by Natural Indigo prepared from Dyer's KnotWeed, J. Korean Society of Clothing and Textiles, 32(12), 1963(2008).

13. C. Oberthur, H. Graf and M. Hamburger, The Content of Indigo Precursors in Isatis tinctoria leaves-A Comparative Study of selected Accessions and Post-harvest Treatment, Phytochemistry, 65, 3261(2004).

14. K. G. Stoker, D. T. Cooke and D. J. Hill, Influence of Light on Natural Indigo Production from Woad (Isatis tinctoria), Plant Growth Regulation, 25, 181(1998). 